\title{
Calculation Model of X-Ray Computed Tomography with Density Assessment Function
}

\author{
S. P. Osipov ${ }^{a, *}$, I. G. Yadrenkin ${ }^{b}$, S. V. Chakhlov ${ }^{a, * *}$, O. S. Osipov ${ }^{c}$, \\ E. Yu. Usachev ${ }^{d}$, and A. A. Manushkin ${ }^{d}$ \\ ${ }^{a}$ Tomsk Polytechnic University, Tomsk, 634028 Russia \\ ${ }^{b}$ Tomsk State University of Architecture and Building, Tomsk, 634003 Russia \\ ${ }^{c}$ Medialooks, Kaliningrad, 236016 Russia \\ ${ }^{d}$ MIREA-Russian Technological University, Moscow, 119454 Russia \\ *e-mail: osip1809@rambler.ru \\ **e-mail: chakhlov@tpu.ru
}

Received November 1, 2020; revised December 2, 2020; accepted December 11, 2020

\begin{abstract}
A calculation model of X-ray computed tomography with a density assessment function in the geometry of a parallel beam has been proposed. The model includes blocks for simulating and correcting sinograms and reconstructing section images. When generating sinograms, the parameters of the test object, source, and recorder of X-ray radiation have been taken into account. Modeling algorithms are implemented in the MathCad system and tested on virtual test objects.
\end{abstract}

Keywords: X-ray sources, X-ray recorders, maximum X-ray energy, X-ray computed tomography, density, effective atomic number

DOI: $10.1134 / \mathrm{S} 1061830921030049$

\section{INTRODUCTION}

In recent decades, there has been a steady increase in interest in X-ray computed tomography (CT) as a method for studying the structure of various test objects with steel dimensions from fractions of millimeters to hundreds of millimeters [1-4]. Prospects for the development of computed tomography are currently associated with measuring linear dimensions, areas, volumes, density, porosity, etc. [5-8]. The tasks of designing computed tomography systems are determined by consumer interests [9-11]. The relevant information is divided into two groups. The first group includes parameters related to the test object: shape, internal structure, and material of fragments. The second group consists of consumer expectations regarding the quality of computed tomography: contrast sensitivity, spatial resolution, the accuracy of the measured parameter assessment, the quality of reproduction of the internal structure by phantoms, and performance. Structurally, computed tomography systems [12] consist of an X-ray source (XRS), an X-ray recorder (XRR), a mechanical system (MS), and a reconstruction algorithm (RA). The solution to the design problem is reduced to a rational assessment of the required parameters of X-ray source, $\mathrm{X}$-ray recorder, mechanical system, and reconstruction algorithms, the selection of the characteristics for the scanning scheme, and the subsequent selection of structural elements of the computed tomography system from instruments and components available on the scientific equipment market.

The components of computed tomography systems are very expensive; therefore, to assess the possibility of solving computed tomography design problems for specific test objects, it is effective to use computational experiments [13-16]. The simplest scanning scheme in computed tomography is the geometry of a narrow parallel beam [12]. For this scheme, the scattering artifact is minimal [12, 17]. The computational model of the computed tomography system consists of forming a set of projections (sinograms) and of image reconstruction. The back-projection algorithm with filtering (BPF) [12, 18, 19] continues to be one of the demanded reconstruction algorithms for computed tomography images; therefore, when developing a computational model of a computed tomography system with a density assessment function, we will use the BPF algorithm.

In addition to solving design problems, computational computed tomography models and software implementing them [20] are necessary for teaching students and postgraduates. It should be noted that, 


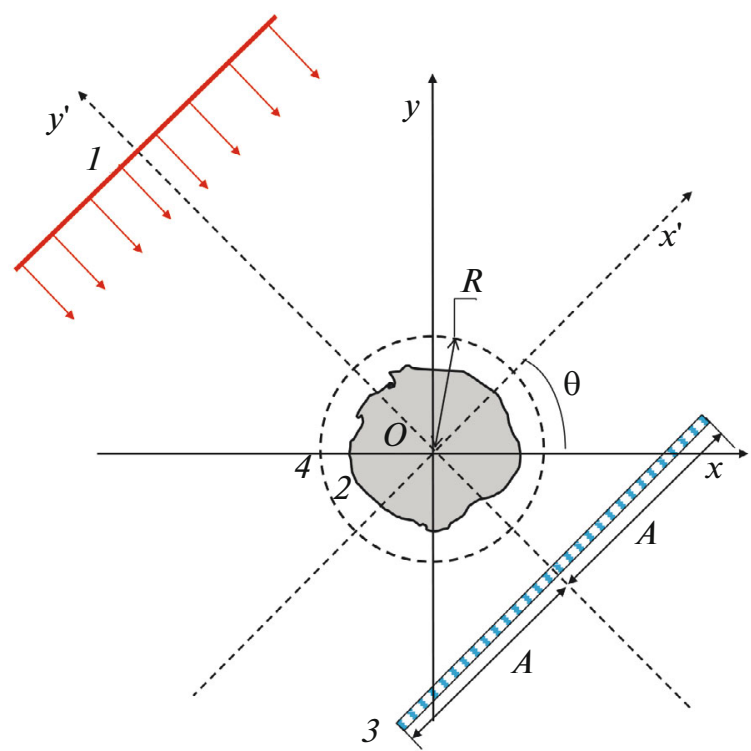

Fig. 1. Scheme of projection formation in X-ray computed tomography: (1) X-ray radiation source; (2) test object; (3) linear $\mathrm{X}$-ray recorder; (4) a circle describing the section of the test object.

with the above taken into account, the MathCad system [21] is ideally suited for the implementation of computational computed tomography models, since its internal language corresponds as much as possible to the natural mathematical language.

\section{COMPUTATIONAL MODEL FOR FORMATION OF SINOGRAMS}

\subsection{Generalized Description of the Object Layer}

Let us introduce a fixed coordinate system $X O Y$. Let $S \subset \mathfrak{R}^{2}$ be the set of points of the test object layer. The test object layer is completely determined if at any point with coordinates $(x, y) \in \mathbf{S}$ the values of the density $\rho(x, y)$ and the effective atomic number (EAN) $Z(x, y)$ are known.

\subsection{Geometric Scheme of Projection Formation}

Figure 1 shows the geometric scheme for the formation of projections in X-ray computed tomography. The origin of the fixed $X O Y$ coordinate system coincides with the test object rotation center. The additional coordinate system $X^{\prime} O Y^{\prime}$ refers to the test object. The coordinates of a point in the $X^{\prime} O Y^{\prime}$ and $X O Y$ coordinate systems are related to each other by a rotation transformation through an angle $\theta$ :

$$
\left\{\begin{array}{l}
x^{\prime}=x \cos \theta+y \sin \theta \\
y^{\prime}=-x \sin \theta+y \cos \theta
\end{array},\left\{\begin{array}{l}
x=x^{\prime} \cos \theta-y^{\prime} \sin \theta \\
y=x^{\prime} \sin \theta+y^{\prime} \cos \theta
\end{array} .\right.\right.
$$

The projection in the computed tomography $P\left(x^{\prime}, \theta\right)$ is the $x^{\prime}$-distribution of the integral of the informative parameter of the test object $p$ along the corresponding $x^{\prime}$-ray, $L\left(x^{\prime}, \theta\right)$. Here $x^{\prime}$ ranges from $-A$ to $A,|A|>R$, and for any point $(x, y) \in \mathbf{S}$ the following condition is satisfied: $x^{2}+y^{2} \leq R^{2}$. Let us write the definition of a projection as a formula,

$$
P\left(x^{\prime}, \theta\right)=\int_{L\left(x^{\prime}, \theta\right)} p\left(y^{\prime}\right) \mathrm{d} y^{\prime} .
$$

For an informative parameter $p$ characterizing the material, the computed tomography uses [22, 23]: the linear radiation attenuation coefficient (LAC) $\mu$, the Hounsfield number, the density $\rho$, and the effective atomic number $Z$. The test object material is most fully characterized by $\rho$ and $Z$. 


\subsection{Generalized Model of Projection Formation in X-ray Computed Tomography}

Models of digital radiographic imaging can be used as the basis for the model of projection formation in computed tomography [24-26]. An expression for how digital signals (DS) from detectors $J_{d}\left(x^{\prime}, \theta\right)$ are connected with the estimate of the projection $P^{*}\left(x^{\prime}, \theta\right)$ has the form

$$
P^{*}\left(x^{\prime}, \theta\right)=-\ln \frac{J_{d}\left(x^{\prime}, \theta\right)-B_{d}\left(x^{\prime}, \theta\right)}{W_{d}\left(x^{\prime}, \theta\right)-B_{d}\left(x^{\prime}, \theta\right)},
$$

here $B_{d}\left(x^{\prime}, \theta\right)$ is the digital signal from the detector with switched off $\mathrm{X}$-ray source (dark signals), and $W_{d}\left(x^{\prime}, \theta\right)$ is the digital signal from the detector without a test object.

For an X-ray source with energy spectrum $f\left(E, E_{\max }\right)$, where $E_{\max }$ is the maximum radiation energy, and a radiation-sensitive element (RSE) of thickness $h_{s}$ operating in integrated recording mode, the ana$\log$ signals (AS) $J\left(E_{\max }, x^{\prime}, \theta\right)$ that correspond to the digital signal $J_{D}\left(E_{\max }, x^{\prime}, \theta\right)$ are estimated using a formula similar to the expression from [25],

$$
\begin{gathered}
J\left(E_{\max }, x^{\prime}, \theta\right)=I\left(E_{\max }, x^{\prime}, \theta\right)+B\left(x^{\prime}\right) \\
=C_{E}\left(x^{\prime}\right) N_{0}\left(E_{\max }, x^{\prime}\right) \int_{0}^{E_{\max }} E_{\mathrm{ab}}\left(E, h_{s}\right) f\left(E, E_{\max }\right) \exp \left(-P_{E}\left(E, x^{\prime}, \theta\right) \varepsilon\left(E, h_{s}\right) \mathrm{d} E+B\left(x^{\prime}\right),\right.
\end{gathered}
$$

where $C_{E}\left(x^{\prime}\right)$ is the factor of conversion of the absorbed radiation energy into the analog signal; $N_{0}\left(E_{\max }, x^{\prime}\right)$ is the number of photons hitting the front surface of the radiation-sensitive element without a test object during projection formation; $E_{\mathrm{ab}}\left(E, h_{s}\right)$ is the average value of energy transmitted to the radiation-sensitive element by a recorded photon with energy $E$; and $\varepsilon\left(E, h_{s}\right)$ is the efficiency of recording. In formula (4) $P_{E}\left(E, x^{\prime}, \theta\right),-A \leq x^{\prime} \leq A$, is the projection for photons with energy $E$ when the informative parameter $p$ is the linear attenuation coefficient of radiation. For a radiation-sensitive element with small dimensions, it is necessary to take into account the leakage and transfer of energy to the environment and to neighboring elements.

The expression for calculating $P_{E}\left(E, x^{\prime}, \theta\right)$ is

$$
P_{E}\left(E, x^{\prime}, \theta\right)=\int_{L\left(x^{\prime}, \theta\right)} \mu\left(E, y^{\prime}\right) \mathrm{d} y^{\prime}=\int_{L\left(x^{\prime}, \theta\right)} m\left(E, Z\left(y^{\prime}\right)\right) \rho\left(y^{\prime}\right) \mathrm{d} y^{\prime},
$$

where $m(E, Z)$ is the mass coefficient of attenuation (MAC) by material with atomic number $Z$ of radiation with energy $E$. Note that $J\left(E_{\max }, x^{\prime}, \theta\right)$ for $P_{E}\left(E, x^{\prime}, \theta\right)=\infty$ coincides with $B\left(x^{\prime}\right)$, and for $P_{E}\left(E, x^{\prime}, \theta\right)=0$, with $W\left(x^{\prime}\right)$.

Analog signals $J\left(E_{\max }, x^{\prime}, \theta\right)$ are transformed into digital signal:

$$
J_{D}\left(E_{\max }, x^{\prime}, \theta\right)=\operatorname{int}\left(\frac{J_{D}\left(E_{\max }, x^{\prime}, \theta\right)}{D}\right), \quad D=\frac{C_{\lim } W\left(x^{\prime}\right)}{2^{k_{A D C}}-1},
$$

here $k_{A D C}$ is the digit capacity of the analog-to-digital converter (ADC); $C_{\text {lim }}, C_{\lim }>1$, is the coefficient limiting the digital signal to the level of $2^{k_{A D C}}-1$ in the case of analog signal fluctuations.

The set of formulas (1)-(6) is a generalized model of the formation of projections in X-ray computed tomography.

\subsection{Formation of Sinograms in X-ray Computed Tomography}

A computed tomography sinogram is a collection of projections. It is an $M \times N$ matrix $\mathbf{P}$, where $M$ is the number of points in the projection and $N$ is the number of projections. The quality of the reconstruction of the sections in the computed tomography is determined by the parameters $M$ and $N$, which are related to the size of the radiation-sensitive element $a$ and the step in the angle $\Delta_{\theta}$ by the relations

$$
N=2 A / a, \quad M=2 \pi / \Delta_{\theta}
$$


The sinogram formation expression is as follows:

$$
\mathbf{P}=\left\{P_{i_{x} i_{\theta}}=P^{*}\left(x_{i x}^{\prime}, \theta_{i_{\theta}}\right) \mid x_{i x}^{\prime}=-A+\frac{a}{2}+a\left(i_{k}-1\right), \quad i_{k}=1 \ldots N ; \quad \theta_{i_{\theta}}=\Delta_{\theta}\left(i_{\theta}-1\right), \quad i_{\theta}=1 \ldots M+1\right\} .
$$

For a visual check of the quality of the initial data in computed tomography, sinograms are presented in graphical form.

\section{RECONSTRUCTION OF CROSS SECTION IMAGES IN X-RAY COMPUTED TOMOGRAPHY METHOD BY BACK PROJECTION WITH FILTERING}

A sinogram is sent to the input of the initial information processing unit. The result of sinogram processing is the distribution of the informative parameter over the test object section. The reason for choosing the BPF method as the reconstruction algorithm of cross section images was noted above.

At the first stage of the algorithm, the initial projections $P^{*}$ are filtered using some filter $h$ :

$$
P^{* *}\left(x^{\prime}, \theta\right)=\int P^{*}(t, \theta) h\left(x^{\prime}-t\right) \mathrm{d} t .
$$

Filters with Ramachandran-Lakshminarayanan (RL) [27] and Shepp-Logan (SL) [28] kernels are most often used as filters in computed tomography.

At the second stage, inverse convolution is implemented, the purpose of which is to estimate the distribution of the information parameter over the test object section $p-p^{*}$ :

$$
p^{*}(x, y)=\int_{0}^{2 \pi} P^{* *}(x \cos \theta+y \sin \theta) \mathrm{d} \theta .
$$

Expressions (9) and (10) make it possible to estimate any function defined on a section for which the complete set of projections (sinogram) is known.

\section{ALGORITHM FOR COMPUTATIONAL MODELING OF FORMATION AND PROCESSING OF SINOGRAMS IN COMPUTED TOMOGRAPHY}

The algorithm for computational modeling of sinograms and reconstruction of images of sections in computed tomography consists of several main blocks: initial data of the computed tomography system; setting of auxiliary functions for attenuation and recording of radiation; description of the test object section; formation of an ideal sinogram; selection of a rational value for the maximum energy of X-ray radiation; the formation of calibration functions of the dependences of the mass thicknesses of the test objects on the their thicknesses in free path lengths (f.p.l.); formation of sinograms; formation of corrected sinograms with their graphic display; and reconstruction of cross section images.

\subsection{Initial Data of X-ray Computed Tomography System}

In accordance with the adopted scheme for the formation of sinograms in X-ray computed tomography, the initial data of the system include: the half-width of the linear X-Ray recorder $A, \mathrm{~mm}$; the transverse dimension of radiation-sensitive element $a, \mathrm{~mm}$; the radiation-sensitive element thickness $h_{s}$, mm; the radiation-sensitive element material; and the angle step $\Delta_{\theta}$, rad.

\subsection{Setting Auxiliary Radiation Attenuation and Recording Functions}

The primary data for this block are borrowed from open libraries on the attenuation of gamma radiation (for example, [29]) and are summarized in tables of mass radiation coefficient of attenuation by substance in the range of atomic number from $Z=1$ (hydrogen) to $Z=82$ (lead). Three vectors correspond to each value of $Z$ : the energy levels of gamma radiation $\mathbf{E}_{n}$; the gamma radiation MAC $\mathbf{m}$; and the average value of absorbed energy $\mathbf{E}_{\mathrm{ab}}$.

This block specifies: the functions $m(E, Z)$ interpolating the dependences of the MAC $m$ on the gamma radiation energy $E$ for substances ranging from hydrogen to lead; the radiation source energy spectrum $f\left(E, E_{\max }\right)$; the energy dependences of the radiation-sensitive element detection efficiency $\varepsilon\left(E, h_{s}\right)$ based on its thickness and material; and an interpolation for the functions $E_{\mathrm{ab}}\left(E, h_{s}\right)$. 


\subsection{Description of Test Object Section}

It was noted above that the test object section is determined by the distributions of the density $\rho(x, y)$ and the effective atomic number $Z(x, y),(x, y) \in \mathbf{S}$. It follows from the analysis of expressions (3)-(6) that the performance of the modeling algorithm depends on the complexity of calculating the energy dependence $P_{E}\left(E, x^{\prime}, \theta\right)$. In [25], the efficiency of replacing the integrals in (4) and (5) by parametric interpolation dependences, for example, on the mass thickness, was noted. The indicated approach can be easily implemented for a test object for which $Z(x, y)=$ const, $(x, y) \in \mathbf{S}$. Let us dwell on test objects of the kind.

In computed tomography, full-scale and virtual phantoms are widely used, which consist of fragments with sections of regular shapes. Most often, circles and squares are used as simple shapes. Here are the descriptions of the test object with such fragments.

\subsubsection{Sections of Fragments in the Form of Circles}

Consider a cylindrical shell with noncontacting cylindrical inclusions of various densities. The axes of the inclusions are parallel to the axis of the cylindrical shell. We will consider sections perpendicular to the shell axis. Such sections are made up of fragments in the form of circles. In this case, the test object section is described by a set of vectors $\mathbf{r}, \mathbf{r} \mathbf{0}, \boldsymbol{\rho}$, and $\boldsymbol{\beta}$ with dimension $n_{0}$ and parameters $x 0$ and $y 0$. Here $\mathbf{r}$ is the vector of the radii of the fragments, $\mathbf{r} \mathbf{0}$ is the vector of the radii of the centers of the fragments, $\boldsymbol{\rho}$ is the vector of the material densities of the fragments, $\boldsymbol{\beta}$ is the vector of the angular coordinates of the centers of the fragments, and $x 0$ and $y 0$ are the coordinates of the displacement of the axis of rotation. Let $i, i=1 \ldots n_{0}$, be the number of the fragment, $i=1$ corresponds to the shell itself, $i=2$ to the axial cavity, and $i=3 \ldots n_{0}$ to the inclusions.

\subsubsection{Sections of Fragments in the Form of Squares}

Thick-walled pipes with a square cross section are a typical representative of test objects with sections of fragments in the form of squares. For the considered test object, all sections of the fragments have the shape of a square. The test object section is determined by a set of vectors $\mathbf{r}, \mathbf{X 0}, \mathbf{Y 0}, \boldsymbol{\rho}$, and $\boldsymbol{\theta}$ with dimension $n_{0}$ and parameters $x 0$ and $y 0$. Here $\mathbf{r}$ is the vector of the radii of the circles inscribed in the fragments; $\mathbf{X 0}$ and $\mathbf{Y 0}$ are the vectors of the coordinates of the centers of the fragments; $\boldsymbol{\rho}$ is the vector of the material density of the fragments; $\boldsymbol{\beta}$ is the vector of the angles of rotation of the fragments, and $x 0$ and $y 0$ are the coordinates of the displacement of the axis of rotation. Fragment numbering is the same as described above: $i=1$ corresponds to the shell; $i=2$ to the axial cavity, and $i=3 \ldots n_{0}$ to shell inclusions.

\subsection{Formation of Ideal Sinograms}

A sinogram is called ideal if the informative parameter is the density $\rho$. To form a sinogram, we will use an approach similar to the additive algorithm for modeling radiographic images [25].

In accordance with [25], the expression for the formation of an ideal sinogram is as follows:

$$
P\left(x^{\prime}, \theta\right)=H_{1}\left(x^{\prime}, \theta\right) \rho_{1}-\sum_{i=2}^{n_{0}}\left(\rho_{i}-\rho_{1}\right) H_{i}\left(x^{\prime}, \theta\right), \quad-A \leq x^{\prime} \leq A, \quad 0 \geq \theta \leq 2 \pi,
$$

here $H_{i}\left(x^{\prime}, \theta\right), i=1 \ldots n_{0}$, are the thicknesses of the test object fragments along the ray passing through a point $x^{\prime}$.

The complexity of calculating ideal sinograms by formula (11) is determined by the shape of the fragments of the test object section. Let us illustrate this for circles and squares.

\subsubsection{Estimation of the beam thicknesses for cross sections of fragments in the form of a circle}

The formula for estimating the thicknesses of the fragments with numbers $i=1 \ldots n_{0}$ is

$$
H_{i}\left(x^{\prime}, \theta\right)=\left\{\begin{array}{l}
2 \sqrt{r_{i}^{2}-\left(x-x 0-r 0_{i} \cos \beta_{i}\right)^{2}},|x-x 0| \leq r 0_{i} \cos \beta_{i} \\
0, \quad|x-x 0|>r 0_{i} \cos \beta_{i}
\end{array},\left\{\begin{array}{l}
x-x 0=x^{\prime} \cos \theta-y^{\prime} \sin \theta \\
y-y 0=x^{\prime} \sin \theta+y^{\prime} \cos \theta
\end{array} .\right.\right.
$$

Expression (12) in combination with (1) is easily implemented in the MathCad system. 


\subsubsection{Estimation of the beam thicknesses for cross sections of fragments in the form of a square}

A square is defined by the coordinates of its center and the coordinates of the corner points. Therefore, at the first stage, the specified coordinates are calculated for the $i$ th fragment. Let there be a local Cartesian coordinate system $X^{\prime \prime} O^{\prime \prime} Y^{\prime \prime}$ anchored at the center of a square fragment, with its axes parallel to the sides of the square. The set of coordinates of corner points of fragments $x_{j i}^{\prime \prime}, y_{j i}^{\prime \prime}, j=1 \ldots 4$, in the corresponding local coordinate systems is described by the expression

$$
\left[\begin{array}{cc}
x_{1 i}^{\prime \prime} & y_{1 i}^{\prime \prime} \\
x_{2 i}^{\prime \prime} & y_{2 i}^{\prime \prime} \\
x_{3 i}^{\prime \prime} & y_{3 i}^{\prime \prime} \\
x_{4 i}^{\prime \prime} & y_{4 i}^{\prime \prime}
\end{array}\right]=\left[\begin{array}{cc}
-r_{i} & r_{i} \\
r_{i} & r_{i} \\
-r_{i} & -r_{i} \\
r_{i} & -r_{i}
\end{array}\right]
$$

Taking into account the rotation of the square fragments by the angles $\beta_{i}$ and the displacement of the centers in the $X O Y$ system, the coordinates of the corner points are calculated by the formula:

$$
\left[\begin{array}{l}
x_{j i} \\
y_{j i}
\end{array}\right]=\left[\begin{array}{cc}
\cos \beta_{i} & -\sin \beta_{i} \\
\sin \beta_{i} & \cos \beta_{i}
\end{array}\right]\left[\begin{array}{c}
x_{j i}^{\prime \prime} \\
y_{j i}^{\prime \prime}
\end{array}\right]+\left[\begin{array}{c}
x 0_{i} \\
y 0_{i}
\end{array}\right] .
$$

The coordinates in the $X^{\prime} O Y^{\prime}$ coordinate system are calculated in a similar way, obtained by rotating the $X O Y$ system relative to the point $O$ by the angle $\theta$ :

$$
\left[\begin{array}{l}
x_{j i}^{\prime} \\
y_{j i}^{\prime}
\end{array}\right]=\left[\begin{array}{cc}
\cos \theta & -\sin \theta \\
\sin \theta & \cos \theta
\end{array}\right]\left[\begin{array}{l}
x_{j i} \\
y_{j i}
\end{array}\right] .
$$

Further, for $i, i=1 \ldots n_{0}$, the matrices $\mathbf{X} \mathbf{Y}_{i}$ are sorted by the first column in ascending order:

$$
\mathbf{X} \mathbf{Y}_{i}=\left[\begin{array}{cc}
x_{1 i}^{\prime} & y_{1 i}^{\prime} \\
x_{2 i}^{\prime} & y_{2 i}^{\prime} \\
x_{3 i}^{\prime} & y_{3 i}^{\prime} \\
x_{4 i}^{\prime} & y_{4 i}^{\prime}
\end{array}\right] \Rightarrow \mathbf{X} \mathbf{Y}_{i}^{*}=\operatorname{csort}(\mathbf{X Y}, 1)=\left[\begin{array}{cc}
x_{1 i}^{*} & y_{1 i}^{*} \\
x_{2 i}^{*} & y_{2 i}^{*} \\
x_{3 i}^{*} & y_{3 i}^{*} \\
x_{4 i}^{*} & y_{4 i}^{*}
\end{array}\right] .
$$

here $\operatorname{csort}\left(\mathbf{X Y} \mathbf{Y}_{i}, 1\right)$ is the sorting procedure by the first column in ascending order.

The estimate of the thickness of the fragments along the beam is found using the formula

$$
H_{i}\left(x^{\prime}, \theta\right)=\left\{\begin{array}{l}
0, \quad\left(x^{\prime}<x_{1 i}^{*}\right) \vee\left(x^{\prime}<x_{4 i}^{*}\right) \\
2 r_{i}, \quad\left(x_{1 i}^{*} \leq x^{\prime} \leq x_{4 i}^{*}\right) \wedge\left(\left(x_{1 i}^{*}=x_{2 i}^{*}\right) \vee\left(x_{3 i}^{*}=x_{4 i}^{*}\right)\right) \\
2 \sqrt{2} r_{i}, \quad x_{2 i}^{*}=x_{3 i}^{*} \\
\left.\frac{y_{2 i}^{*}-y_{1 i}^{*}}{x_{2 i}^{*}-x_{1 i}^{*}}\left(x^{\prime}-x_{1 i}^{*}\right) \mid-\frac{y_{3 i}^{*}-y_{1 i}^{*}}{x_{3 i}^{*}-x_{1 i}^{*}}\left(x^{\prime}-x_{1 i}^{*}\right)\right), \quad\left(x_{1 i}^{*} \leq x^{\prime}<x_{2 i}^{*}\right) \wedge\left(x_{1 i}^{*} \neq x_{2 i}^{*}\right) \\
\left.\frac{y_{3 i}^{*}-y_{1 i}^{*}}{x_{3 i}^{*}-x_{1 i}^{*}}\left(x^{\prime}-x_{1 i}^{*}\right)+y_{1 i}^{*}-\frac{y_{4 i}^{*}-y_{2 i}^{*}}{x_{4 i}^{*}-x_{2 i}^{*}}\left(x^{\prime}-x_{2 i}^{*}\right)-y_{2 i}^{*}\right), \quad\left(x_{2 i}^{*} \leq x^{\prime}<x_{3 i}^{*}\right) \wedge\left(x_{2 i}^{*} \neq x_{3 i}^{*}\right) \\
\left.\frac{y_{4 i}^{*}-y_{2 i}^{*}}{x_{4 i}^{*}-x_{2 i}^{*}}\left(x^{\prime}-x_{2 i}^{*}\right)+y_{2 i}^{*}-\frac{y_{4 i}^{*}-y_{3 i}^{*}}{x_{4 i}^{*}-x_{3 i}^{*}}\left(x^{\prime}-x_{3 i}^{*}\right)-y_{3 i}^{*}\right), \quad\left(x_{3 i}^{*} \leq x^{\prime}<x_{4 i}^{*}\right) \wedge\left(x_{3 i}^{*} \neq x_{4 i}^{*}\right) .
\end{array}\right.
$$


Expressions (13)-(17) make it possible to estimate the beam thicknesses of sections of fragments in the form of a square for any values of $x^{\prime}$ and $\theta$.

The above formulas (12)-(17) are the basis of algorithms for the formation of ideal sinograms for sections of fragments in the form of a circle and a square.

\subsection{Selecting the Maximum X-ray Energy}

The choice of the maximum energy of X-ray radiation has a significant effect on the quality of reconstruction of images of cross sections in computed tomography [30]. The equation for selection of $E_{\max }$ is similar to the equation specified in [30],

$$
\ln \frac{\int_{0}^{E_{\max }} E_{\mathrm{ab}}\left(E, h_{s}\right) f\left(E, E_{\max }\right) \varepsilon\left(E, h_{s}\right) \mathrm{d} E}{\int_{0}^{E_{\max }} E_{\mathrm{ab}}\left(E, h_{s}\right) f\left(E, E_{\max }\right) \exp \left(-m(E, Z) \max _{x^{\prime} \in[-A, A], \theta \in[0,2 \pi]} P\left(x^{\prime}, \theta\right) \varepsilon\left(E, h_{s}\right) \mathrm{d} E\right.}=2 .
$$

The right-hand side of Eq. (18) can be decreased or increased. The value $P_{\max }=\max _{x^{\prime} \in[-A, A], \theta \in[0,2 \pi]} P\left(x^{\prime}, \theta\right)$ is equal to the maximum mass thickness of the test object.

\subsection{Formation of Calibration Functions, Dependences of the Mass Thicknesses of Test Objects on Their Thicknesses in Free Path Lengths}

It follows from the definition of an ideal sinogram that it is necessary to estimate the mass thickness of the test objects for all beams. For this, the results of a full-scale or computational experiment are used to determine the dependences of the X-ray attenuation coefficients on the mass thickness $\rho H$.

We will use the algorithm for the formation of calibration functions for EAN -homogeneous test objects given in [31]. A stepped object with steps of thickness from $(\rho H)_{1}=\rho H_{\text {min }}=0 \mathrm{~g} / \mathrm{cm}^{2}$ to $(\rho H)_{l}=\rho H_{\max }=P_{\max } \mathrm{g} / \mathrm{cm}^{2}$ acts as a test object. The formula of relationship between the estimate of the thickness of the test object in f.p.l. $Y$ and the mass thickness $\rho H$ has the form

$$
Y\left(E_{\max }, \rho H\right)=-\ln \frac{\operatorname{int} C_{E} N_{0} \int_{0}^{E_{\max }} E_{\mathrm{ab}}\left(E, h_{s}\right) f\left(E, E_{\max }\right) \exp (-m(E, Z) \rho H) \varepsilon\left(E, h_{s}\right) \mathrm{d} E / D}{\operatorname{int} C_{E} N_{0} \int_{0}^{E_{\max }} E_{\mathrm{ab}}\left(E, h_{s}\right) f\left(E, E_{\max }\right) \varepsilon\left(E, h_{s}\right) \mathrm{d} E / D} .
$$

The table $\mathbf{B}=\left((\rho H)_{n}, Y\left(E_{\max },(\rho H)_{n}\right)\right), n=1 \ldots l$. is fed to the input of the algorithm for forming the calibration functions. The analytical function $F$ is constructed on the basis of table $\mathbf{B}$

$$
\rho H=F(Y) \text {. }
$$

Formula (20) allows one to evaluate ideal sinograms by real ones.

\subsection{Formation of Real Sinograms and Their Graphic Display}

The input of the algorithm for generating sinograms based on expressions (3)-(8) receives an ideal sinogram. This sinogram for the test object, consisting of a finite number of fragments with different densities, is described by expression (11). For a detailed implementation of the algorithm being carried out for specific shapes of fragments of sections of the test object, for example, circles and squares, see (12)-(17). As a result of applying the algorithm to a specific test object, an ideal sinogram $\mathbf{P}=\left\{P_{i_{x} i_{\theta}}=P\left(x_{i_{x}}^{\prime}, \theta_{\mathrm{i}_{\theta}}\right): i_{x}=1 \ldots N ; i_{\theta}=1 \ldots M+1\right\}$ is formed 
Relationship between the ideal sinogram $\mathbf{P}$ and the real sinogram $\mathbf{P}=\left\{P_{i_{x} i_{\theta}}^{*}: i_{x}=1 \ldots N ; i_{\theta}=1 \ldots M+1\right\}$ is described by the expression

$$
P_{i_{x} i_{\theta}}^{*}=-\ln \frac{\operatorname{int}\left(\int_{0}^{E_{\max }} E_{\mathrm{ab}}\left(E, h_{s}\right) f\left(E, E_{\max }\right) \exp \left(-m(E, Z) P_{i_{x} i_{\theta}}\right) \varepsilon\left(E, h_{s}\right) \mathrm{d} E / D\right)}{\operatorname{int}\left(\int_{0}^{E_{\max }} E_{\mathrm{ab}}\left(E, h_{s}\right) f\left(E, E_{\max }\right) \varepsilon\left(E, h_{s}\right) \mathrm{d} E / D\right)} .
$$

The synogram $\mathbf{P} *$ is converted into a grayscale image $\mathbf{G}^{*}$ as follows:

$$
G_{i_{x_{\theta}} i_{\theta}}^{*}=255-255 \operatorname{int}\left(\frac{P_{i_{x} i_{\theta}}^{*}-\min \left(\mathbf{P}^{*}\right)}{\max \left(\mathbf{P}^{*}\right)-\min \left(\mathbf{P}^{*}\right)}\right) .
$$

The graphical representation of the sinogram is necessary not only for the visual presentation of information in one image, but also for the analysis of possible distortions during scanning or computational modeling.

\subsection{Correction of Sinograms}

In sinograms, the test object thicknesses along the rays are measured in f.p.1., which makes it impossible to accurately estimate the density distribution over the cross section of the object by the computed tomography method. Correction of sinograms is reduced to recalculation of the thickness of the test object along the rays in the f.p.l. into mass thicknesses of test object along the corresponding rays.

The sinogram $\mathbf{P}^{*}$ is corrected in accordance with the transformation (20). The expression for the formation of the final sinogram $\mathbf{P}_{C}$ is

$$
\mathbf{P}_{C}=\left\{P_{C i_{x} i_{\theta}}=F\left(P_{i_{x} i_{\theta}}^{*}\right): i_{x}=1 \ldots N ; i_{\theta}=1 \ldots M+1\right\} .
$$

The graphical image of the sinogram $\mathbf{P}^{*}$ is formed similar to formula (22).

\subsection{Reconstruction of Section Images by the Inverse Convolution Method with Filtering}

The algorithm and the program for reconstructing images of test object cross sections in computed tomography are independent and linked with each other by a text file. A sinogram is written in a text file, for example, the $\operatorname{Pr}$ matrix. The file name contains information about the radiation-sensitive element size $a$.

After reading the matrix $\operatorname{Pr}$, the number of detectors in the line $N$, and the number of projections $M$ are determined:

$$
N=\operatorname{rows}(\mathbf{P r}), \quad M=\operatorname{cols}(\mathbf{P r}) .
$$

here rows $(\mathbf{P r})$ and cols $(\mathbf{P r})$ are the numbers of rows and columns in the matrix $\mathbf{P r}$.

Next, filters with RL and SL kernels are set.

\subsubsection{Ramachandran-Lakshminarayanan and Sheppa-Logan Filters}

The Ramachandran-Lakshminarayanan filter $f_{\mathrm{RL}}$ is defined by the formula [27]

$$
f_{\mathrm{RL} i}=\left\{\begin{array}{l}
\frac{1}{4 a^{2}}, \quad i=0, \\
0, \quad i=2 k, \quad k= \pm 1, \pm 2, \ldots \\
\frac{-1}{\pi^{2} a^{2} i^{2}}, \quad i=2 k-1, \quad k= \pm 1, \pm 2, \ldots
\end{array}, \quad i=-N h \ldots N h, \quad N h=\operatorname{int}\left(\frac{N}{2}\right) .\right.
$$


The expression for the Shepp-Logan filter $f_{\mathrm{ShL}}$ has the form [28]

$$
f_{\mathrm{ShL} i}=\frac{1}{\pi^{2} a^{2}}\left(\frac{1}{1-4 i^{2}}\right), \quad i=-N h \ldots N h, \quad N h=\operatorname{int}\left(\frac{N}{2}\right) .
$$

\subsubsection{Filtering Initial Projections}

The filtering of the initial projections $\mathbf{P r}$ is carried out in accordance with expression (8), which in discrete form looks as follows:

$$
\operatorname{Prf}_{i_{x} i_{\theta}}=a \sum_{i_{f}=-N x}^{N x} \operatorname{Pr}_{i_{f} i_{\theta}}\left\{\begin{array}{l}
f_{i_{x}-i_{f}}, \quad-N_{x} \leq i_{x}-i_{f}<N_{x} \\
0, \quad\left(i_{x}-i_{f}<-N_{x}\right) \vee\left(i_{x}-i_{f} \geq N_{x}\right)
\end{array} .\right.
$$

In expression (27), a filter with the RL kernel $f_{\mathrm{RL}}$ (26) or a filter with the SL kernel $f_{\mathrm{ShL}}$ (27) or their modifications are used as the filter $f$.

\subsubsection{Back Projection}

The filtered Prf projection is input into the back projection unit. In accordance with formula (9), the estimate of the informative parameter at the point of the section $(x, y)$ is the integral over all rays passing through the marked point.

In the case under consideration, the informative parameter is the density $\rho$; therefore, the estimate for the density $\rho$ at a point with coordinates $(x, y)$ is found by the formula:

$$
\tilde{\rho}(x, y) \approx h_{\theta} \sum_{i_{\theta}=1}^{M} \operatorname{Prf}^{*}\left(x \cos \theta_{i_{\theta}}+y \sin \theta_{i_{\theta}}, \theta_{i_{\theta}}\right) .
$$

The function $\operatorname{Prf}^{*}\left(x^{*}, \theta_{i_{\theta}}\right)$ for a fixed value of $i_{\theta}$ is an interpolation of the discrete dependence of $\operatorname{Prf}_{i_{x} i_{\theta}}$ on $x_{i_{x}}^{\prime}$.

\subsubsection{Cross Section Imaging}

At the first stage, a digital image is formed $\tilde{\rho}$, representing the distribution of the estimate of the informative parameter (density) over the test object section.

Let the image have the shape of a rectangle with dimensions of $N_{x} \times N_{y}$ pixels. Then the matrix $\tilde{\rho}=\left\{\rho_{i_{x} i_{y}}, i_{x}=1 \ldots N_{x}, i_{y}=1 \ldots N_{y}\right\}$ is described by the expression

$$
\tilde{\rho}=\left\{\begin{array}{ll}
\tilde{\rho}\left(x_{i_{x}}, y_{i_{y}}\right), & x_{i_{x}}^{2}+y_{i_{y}}^{2} \leq A^{2} \\
0, & x_{i_{x}}^{2}+y_{i_{y}}^{2}>A^{2}
\end{array}, \quad x_{i_{x}}=-A+\frac{2 A}{N_{x}-1}\left(i_{x}-1\right), \quad y_{i_{y}}=-A+\frac{2 A}{N_{y}-1}\left(i_{y}-1\right)\right\} .
$$

At the second stage, based on the image $\tilde{\rho}$, the corresponding grayscale image $\mathbf{G}_{\boldsymbol{\rho}}$ is formed according to a formula similar to (23).

The aim of this work is to construct a computational model of X-ray computed tomography with estimation of density over the test object cross section; therefore, the image $\tilde{\rho}$ is processed in order to determine the deviations of the density estimates from their real values.

\section{EXAMPLES OF SIMULATION AND PROCESSING OF SINOGRAMS IN COMPUTED TOMOGRAPHY}

To illustrate the capabilities of the algorithms for modeling sinograms, their correction, and reconstruction of density distributions over test object sections, we selected objects with the sections of fragments in the shape of a circle and a square. All test object fragments are homogeneous in EAN $(Z=13)$ but vary in density.

The developed algorithms are implemented in the MathCad 15 system in the form of programs for generating sinograms and image reconstruction. The program for generating sinograms is universal, with the exception of the test object description block with the calculation of its thickness by rays. The reconstruc- 


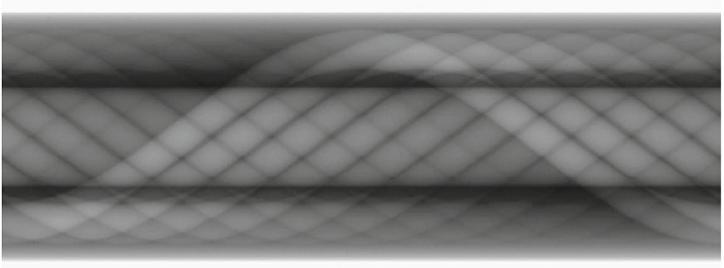

(a)

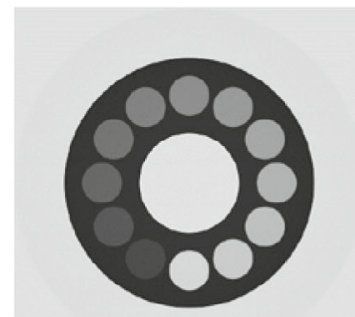

(b)

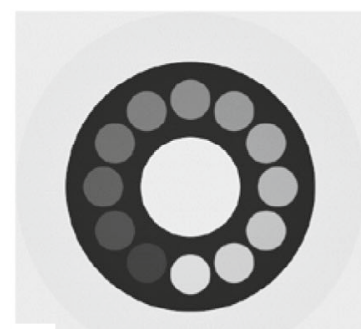

(c)

Fig. 2. Object with sections of fragments in the form of circles: (a) sinogram; (b) reconstruction (RL filter); (c) reconstruction (SL filter).

tion program is universal; it contains switchable blocks for calculating the coefficients of the RL and SL filters. The above programs are connected through a text file corresponding to the Pr sinogram.

The array of X-ray detectors has a size of $2 A=70 \mathrm{~mm}$. The radiation-sensitive element material is cadmium tungstate, the radiation-sensitive element transverse dimension is $a=0.1 \mathrm{~mm}$, and the radiationsensitive element thickness is $h_{s}=0.3 \mathrm{~mm}$. A total of $N_{0}=10^{6}$ photons fall on the front surface of each radiation-sensitive element.

\subsection{Object with Sections of Fragments in the Form of a Circle}

The test object section is characterized by the following parameters: the outer radius of the thickwalled cylindrical shell $r_{1}=25 \mathrm{~mm}$; the radius of the inner cylindrical cavity $r_{2}=10 \mathrm{~mm}$; the radii of local shell fragments, $r_{3}=r_{4}=\ldots=r_{14}=4 \mathrm{~mm}$; the radial coordinates of the centers of the fragments are $r 0_{1}=r 0_{2}=0 \mathrm{~mm}, r 0_{3}=r 0_{4}=\ldots=r 0_{14}=17.5 \mathrm{~mm}$; the angular coordinates of the centers of the fragments $\beta_{1}=\beta_{2}=0, \beta_{i}=\pi(i-3) / 6, i=3 \ldots 14$; and the material density of the fragments $\rho_{1}=2.7 \mathrm{~g} / \mathrm{cm}^{3}$, $\rho_{2}=0 \mathrm{~g} / \mathrm{cm}^{3}, \rho_{i}=0.2(i-2) \mathrm{g} / \mathrm{cm}^{3}, i=3 \ldots 14$.

The number of projections is $M=1440$. The maximum value of the mass thickness along the rays for

the object under consideration is $P_{\max }=11.7 \mathrm{~g} / \mathrm{cm}^{2}$. The rational value for the maximum energy of X-ray radiation was chosen based on condition (19) at $E_{\max }=400 \mathrm{keV}$.

Figure 2 shows the images of the sinogram for the section of the analyzed object and the images of the section reconstructed by the BPF method.

The sinogram formation time for the object under study on a laptop with an Intel (R) Core (TM) i5-83000H $\mathrm{CPU}$ with a frequency of $2.30 \mathrm{GHz}$ does not exceed $85 \mathrm{~s}$. The image reconstruction time with the RL filter does not exceed 11 minutes, and with the SL filter it is 10.3 minutes. The specified performance of the programs is sufficient for use in the educational process and for a detailed analysis of the influence of the parameters of $X$-ray computed tomography systems on the quality of image reconstruction in the imaging mode. The reconstructed images for the RL and SL filters are close to each other in quality.

To assess the quality of modeling of computed tomography systems operating in the mode of measuring density distributions over the test object section, we determine the dependence of the density $\tilde{\rho}_{c}$ on the angle $\varphi$ along a circle with a radius of $r_{c}=r 0_{3}=17.5 \mathrm{~mm}$ centered at the geometric center of the section. The formula for estimating the dependence $\tilde{\rho}_{c}(\varphi)$ has the form

$$
\tilde{\rho}_{c}=\tilde{\rho}\left(r_{c} \cos \varphi, r_{c} \sin \varphi\right) .
$$

Figure 3 shows copies of the graphs produced by the MathCad program for dependences $\rho_{c}(\varphi)$ with the employed RL and SL filters.

It can be concluded from the analysis of the graphs shown in Fig. 3 that the density distribution over the cross section of the considered test object obtained by the computed tomography method for reconstruction using the back projection method with RL and SL filtering is highly accurate. 

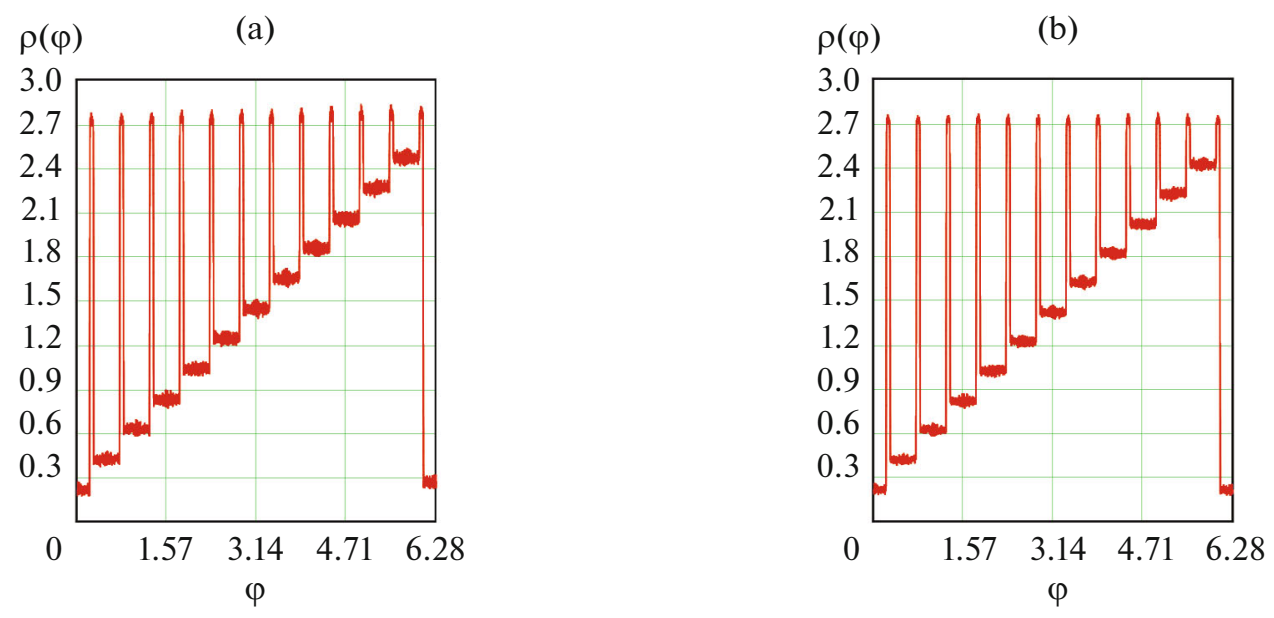

Fig. 3. Angular dependences $\tilde{\rho}_{c}(\varphi)$ for TO with sections of fragments in the form of circles: (a) filter with RL kernel; (b) filter with SL kernel.
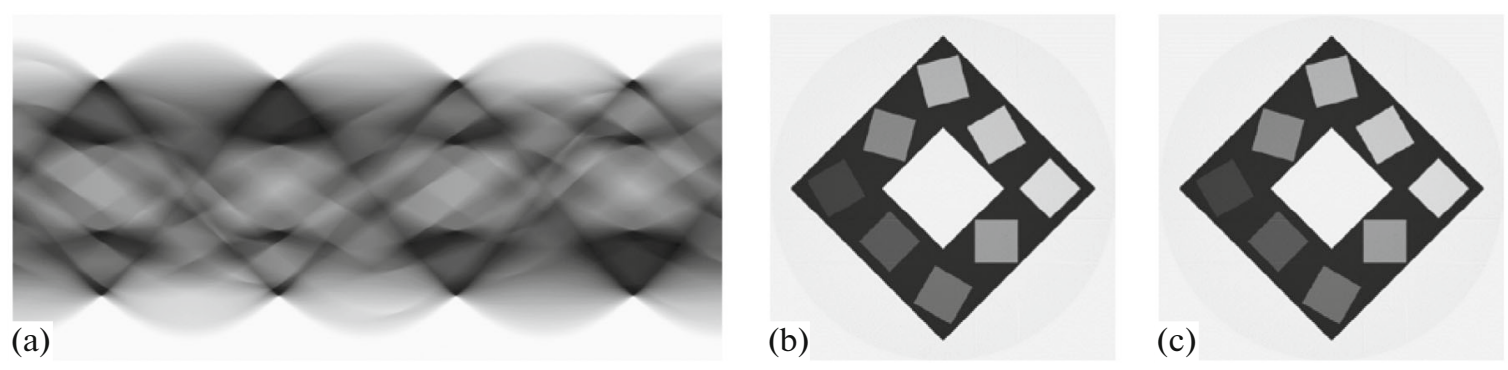

Fig. 4. Object with sections of fragments in the form of squares: (a) sinogram; (b) reconstruction (RL filter); (c) reconstruction (SL filter).

\subsection{Object with Sections of Fragments in the Form of a Square}

The sections of the considered test objects are characterized by the following parameters: the radius of the circle inscribed in a thick-walled shell with a square section $r_{1}=25 \mathrm{~mm}$; the radius of the circle inscribed in the inner cavity with a square section $r_{2}=10 \mathrm{~mm}$; the radii of the circles inscribed in the sections of square fragments located inside the shell, $r_{3}=r_{4}=\ldots=r_{10}=5 \mathrm{~mm}$; the coordinates of the centers of the fragments $X 0_{1}=Y 0_{1}=X 0_{2}=Y 0_{2}=0 \mathrm{~mm}, X 0_{3}=Y 0_{3}=-17.5 \mathrm{~mm}, X 0_{4}=-17.5 \mathrm{~mm}, \quad Y 0_{4}=0 \mathrm{~mm}$, $X 0_{5}=-17.5 \mathrm{~mm}, Y 0_{5}=-17,5 \mathrm{~mm}, X 0_{6}=0 \mathrm{~mm}, Y 0_{6}=-17.5 \mathrm{~mm}, X 0_{7}=0 \mathrm{~mm}, Y 0_{7}=17.5 \mathrm{~mm}$, $X 0_{8}=17.5 \mathrm{~mm}, Y 0_{8}=-17.5 \mathrm{~mm}, X 0_{9}=17.5 \mathrm{~mm}, Y 0_{9}=0 \mathrm{~mm}, X 0_{10}=17.5 \mathrm{~mm}, Y 0_{10}=17.5 \mathrm{~mm}$; the angles of rotation of the fragments $\beta_{1}=\beta_{2}=0, \beta_{i}=\pi(i-3) / 12, i=3 \ldots 14$; and the material density of the fragments $\rho_{1}=2.7 \mathrm{~g} / \mathrm{cm}^{3}, \rho_{2}=0 \mathrm{~g} / \mathrm{cm}^{3}, \rho_{i}=0.3(i-2) \mathrm{g} / \mathrm{cm}^{3}, i=3 \ldots 10$.

The number of projections is $M=1440$. The maximum mass thickness along the rays for the object under consideration is $P_{\max }=13.8 \mathrm{~g} / \mathrm{cm}^{2}$. The rational value for the maximum X-ray radiation energy was chosen based on condition (19) at $E_{\max }=450 \mathrm{keV}$.

Figure 4 shows the images of the sinogram for the cross section of the investigated test object and the results of reconstruction. Filters with RL and SL kernels were used in the BPF algorithm.

The sinogram simulation time for the analyzed test object on a laptop with an Intel (R) Core (TM) i5-83000H CPU with a frequency of $2.30 \mathrm{GHz}$ does not exceed $129 \mathrm{~s}$. The time of forming images of cross sections with the RL filter does not exceed 15 minutes, and with the SL filter-14 minutes. This performance of the programs is sufficient for use in the educational process and for a thorough analysis of the influence of the parameters of X-ray computed tomography systems on the quality of visualization of cross section images. The rendered images for the RL and SL filters are close to each other in quality. 

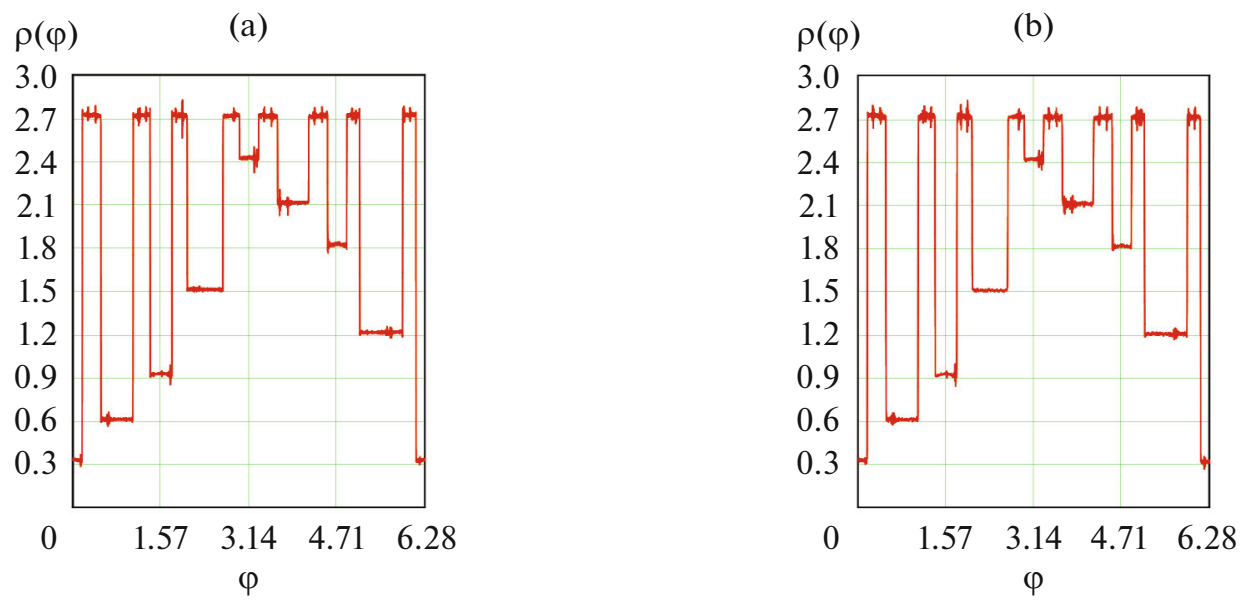

Fig. 5. Angular dependences $\tilde{\rho}_{c}(\varphi)$ for test object with sections of fragments in the form of squares: (a) filter with RL kernel; (b) filter with SL kernel.

For the considered test object, the accuracy of measuring the dependence of density $\tilde{\rho}_{c}(\varphi)$ from the angle $\varphi$ is estimated along the line connecting the centers of the local fragments of the shell. Figure 5 shows copies of the graphs produced by the MathCad program for dependences $\tilde{\rho}_{c}(\varphi)$ in relation to the investigated test object for RL and SL filters. Comparison of the graphs confirms the conclusion about the high accuracy of estimating the density distribution over the cross section of the analyzed object by the computed tomography method for reconstruction by the BPF method with RL and SL filters.

\section{DISCUSSION}

The reconstructions of the internal structure of the test object with sections of fragments in the form of circles and similar objects from [32] visually have the same quality level. We come to the same conclusion as a result of comparing Figs. $4 \mathrm{~b}$ and $4 \mathrm{c}$ with the reconstructed images of the cross sections of a universal phantom for computed tomography with similar fragments [33]. In both cases, the accuracy of density estimation for similar fragment materials is not inferior to the corresponding experimental values from [32]. From the above, we can draw a conclusion about the adequacy of the developed computational model for test objects made of materials homogeneous in EAN with fragments with sections in the form of a circle and a square.

Let us briefly discuss the approaches to estimating the density distribution by the computed tomography method for test objects with more complex sections of fragments or with fragments differing in EAN.

\subsection{Cross Section of an Object with Complex Shaped Fragments Made of Materials Homogeneous in Effective Atomic Number}

The specificity of the test object affects exclusively the process of forming sinograms.

The approach in [25] can be used to modify the block for describing the test object sections. This approach is based on expression (11) using information about the shapes, sizes, and materials of fragments of the test object section. In [25], the idea is also mentioned, according to which, based on the image of a fragment with small pixels, images with pixels of much larger size are constructed. Let us modify this idea by changing the method of obtaining the initial images of fragments.

The shape and size of technical objects are known and, as a rule, documented with drawings. In accordance with the above, it is sufficient to consider the principle of describing one fragment of a complex shape. Let some continuous closed curve $\mathbf{W}$ be given on the plane; the curve is the outer boundary of some plane figure (fragment) $\boldsymbol{\Phi}$. The sectional drawing of a fragment can be presented on paper or in electronic form. The drawing is converted into a digital image in *.bmp format with the highest possible resolution. The digital image is transformed into an ideal sinogram, which is processed according to the algorithm described above.

Figure 6 shows a true image of the cross section of an aluminum test object in the form of a star with sixteen rays, the sinogram, and the results of reconstruction. The radius of the circumscribed circle is 


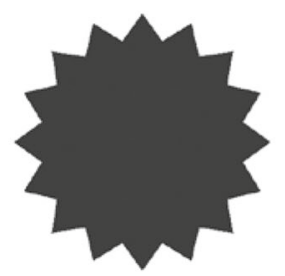

(a)

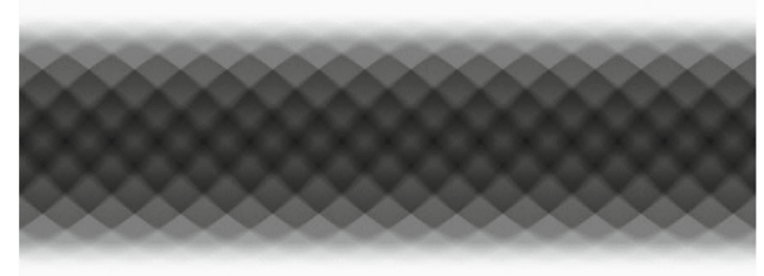

(b)

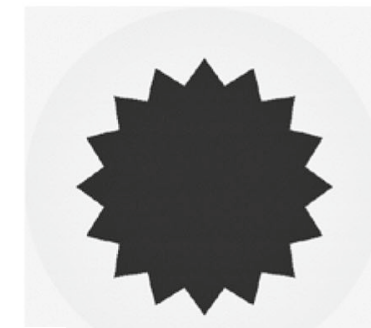

(c)

Fig. 6. Object with fragment sections in the form of a star: (a) object section image; (b) sinogram; (c) reconstruction (SL filter).

$R=25 \mathrm{~mm}$, and the radius of the inscribed circle is $r=20 \mathrm{~mm}$. The number of projections is $M=1440$. The number of detectors in the array is $N=700$. The maximum value of the mass thickness along the rays for the object under study is $P_{\max }=13.5 \mathrm{~g} / \mathrm{cm}^{2}$, and $E_{\max }=450 \mathrm{keV}$.

The time to form the sinogram is approximately an hour, with most of it is spent on evaluating the ideal projections. The reconstruction time is close to $13 \mathrm{~min}$. The visual high quality of the reconstruction should be noted. The density estimation error does not exceed $2 \%$. In general, the quality of the reconstruction is close to the quality of the experimental images of the cross sections given in [34] for the gearwheel test object.

\subsection{Sections of objects with fragments differing in $Z$}

The presence of fragments with materials with different EANs in the test object leads to a significant shift in the density estimates. To eliminate the noted shift, the dual-energy methods (DEM) are used in computed tomography. The dual-energy methods in computed tomography are based on transillumination of the test object with gamma or X-rays with two energies $[35,36]$ and the subsequent estimation of the EAN and density distributions. The maximum productivity is achieved by the implementation of the DEM [37], in which monoenergetic gamma radionuclides are used as radiation sources.

The energy spectra of monoenergetic radionuclides are described by $\delta$-functions. In accordance with this and formulas (4) and (5), the system connecting the parameters of the test object and the projection for the energies of gamma radiation $E_{1}$ and $E_{2}$ has the following form:

$$
\begin{aligned}
& P\left(E_{1}, x^{\prime}, \theta\right)=\int_{L\left(x^{\prime}, \theta\right)} m\left(E_{1}, Z\left(y^{\prime}\right)\right) \rho\left(y^{\prime}\right) \mathrm{d} y^{\prime}=P_{1}\left(x^{\prime}, \theta\right) ; \\
& P\left(E_{2}, x^{\prime}, \theta\right)=\int_{L\left(x^{\prime}, \theta\right)} m\left(E_{2}, Z\left(y^{\prime}\right)\right) \rho\left(y^{\prime}\right) \mathrm{d} y^{\prime}=P_{2}\left(x^{\prime}, \theta\right) .
\end{aligned}
$$

Two sinograms $\mathbf{P}_{1}$ and $\mathbf{P}_{2}$ are formed in the program for simulating sinograms. The LAC of radiation acts as an informative parameter in the reconstruction of cross section images, but the representation of the projections in the form (31) allows the necessary modification of the algorithm in order to simultaneously estimate the distributions of density $\tilde{\rho}(x, y)-\tilde{\boldsymbol{\rho}}$ and EAN $\tilde{Z}(x, y)-\tilde{\mathbf{Z}}$. As a result of the reconstruction, the following distributions are estimated:

$$
\begin{aligned}
& \tilde{m}\left(E_{1}, \tilde{Z}(x, y)\right) \tilde{\rho}(x, y)=\tilde{\mu}\left(E_{1}, x, y\right) ; \\
& \tilde{m}\left(E_{2}, \tilde{Z}(x, y)\right) \tilde{\rho}(x, y)=\tilde{\mu}\left(E_{2}, x, y\right) .
\end{aligned}
$$

Estimation of the EAN distribution $\tilde{Z}(x, y)$ is carried out by a formula close to [36],

$$
\tilde{Z}(x, y)=G(Q(x, y))=G\left(\tilde{m}\left(E_{1}, \tilde{Z}(x, y)\right) / \tilde{m}\left(E_{2}, \tilde{Z}(x, y)\right)\right) .
$$

The function $G$ is determined at the stage of calibration tests on samples made of various materials that differ in EAN. Consider a test object with sections of fragments in the form of circles with variation in density and EAN. The fragment sizes and parameters are identical to those in Sec. 4.1. The local fragments in the shell are divided into six groups: $Z=6\left(\rho=1.5 \mathrm{~g} / \mathrm{cm}^{3} ; 2.2 \mathrm{~g} / \mathrm{cm}^{3}\right) ; Z=9\left(\rho=1.5 \mathrm{~g} / \mathrm{cm}^{3} ; 2.2 \mathrm{~g} / \mathrm{cm}^{3}\right)$; $Z=17\left(\rho=2 \mathrm{~g} / \mathrm{cm}^{3} ; 2.5 \mathrm{~g} / \mathrm{cm}^{3}\right) ; Z=22\left(\rho=2 \mathrm{~g} / \mathrm{cm}^{3} ; 3 \mathrm{~g} / \mathrm{cm}^{3}\right) ; Z=26\left(\rho=3 \mathrm{~g} / \mathrm{cm}^{3} ; 5 \mathrm{~g} / \mathrm{cm}^{3}\right)$; and $Z=29\left(\rho=3 \mathrm{~g} / \mathrm{cm}^{3} ; 5 \mathrm{~g} / \mathrm{cm}^{3}\right)$. The scan parameters are identical to the example in Sec. 4.1 . 
(a)

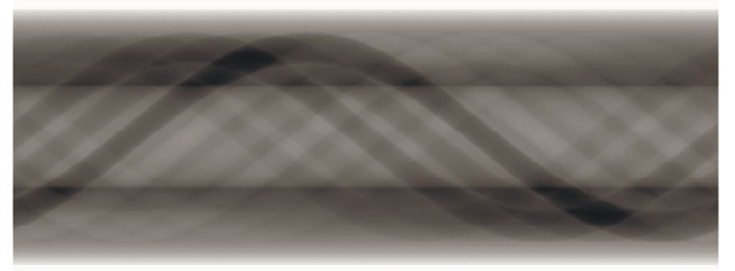

(c)

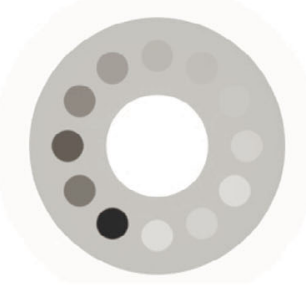

(d)

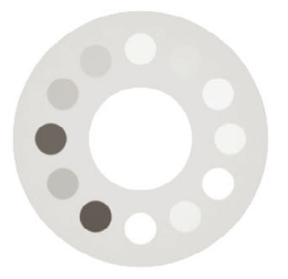

(b)

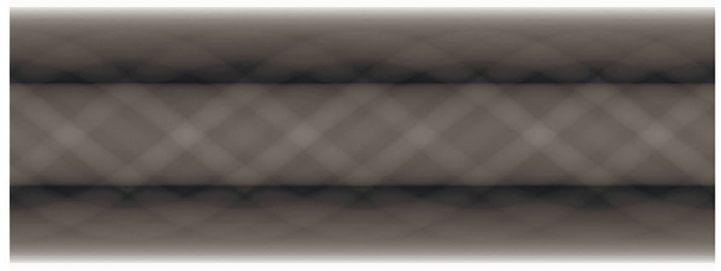

(e)

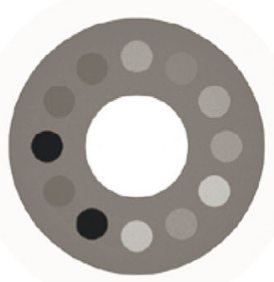

(f)

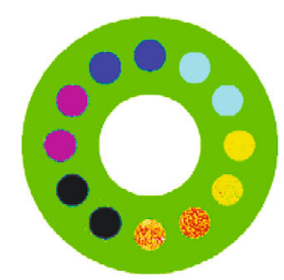

Fig. 7. Test object section with fragments differing in material EAN: (a) sinogram for energy $E_{1}$; (b) sinogram for energy $E_{2}$; (c) LAC reconstruction for energy $E_{1}$; (d) LAC reconstruction for energy $E_{2}$; (e) reconstruction of $\rho$; (f) reconstruction $Z$.

Figure 7 shows the sinograms for the $\gamma$-radiation energies $E_{1}=100 \mathrm{keV}$ and $E_{2}=225 \mathrm{keV}$, the results of preliminary reconstruction, and the estimates of the distributions of EAN and density. The following palette was applied: $Z=6$ red; $Z=9$ yellow; $Z=13$ green; $Z=17$ light blue; $Z=22$ blue; $Z=26$ bright purple; and $Z=29$ black.

The formation time of two sinograms for monoenergetic radiation sources on the aforementioned laptop does not exceed two minutes. The total reconstruction time is close to half an hour. The data presented in Fig. 7 illustrate the possibility of simultaneous estimation of the density and EAN distributions over the test object section. The errors in the estimates of the density and EAN of the materials of the test object fragments obtained as a result of the computational experiment do not exceed the levels of errors in the estimates of the density and the EAN of the materials of the fragments of the test object fragments with a similar structure [37].

\section{CONCLUSIONS}

A computational model of X-ray computed tomography with the function of estimating the density distribution over the cross section of the test object in the parallel beam geometry has been presented.

The modeling algorithm consists of a block for generating sinograms and a block for reconstructing the images of object sections. The mathematical model is implemented in the MathCad software for engineering calculations. The performance of the algorithm is illustrated for objects with fragments with sections in the shape of circles and squares and are homogeneous in effective atomic number of material. The possibility of studying objects with complex shaped cross sections of fragments is demonstrated using the example of a multibeam star. The proposed modeling algorithm is supplemented by the dual energy method, which allows one to simultaneously estimate the distributions of density and effective atomic number over the cross section of the object. The efficiency of the dual-energy method is illustrated by the example of objects with fragments with sections in the form of circles with varying density and effective atomic number of the fragment material. The developed computational models and software implementing them in the MathCad system are intended for the selection and evaluation of the parameters of X-ray computed tomography systems with the function of density estimation over the cross section of the object under study at the design stage and for use in the educational process.

\section{OPEN ACCESS}

This article is licensed under a Creative Commons Attribution 4.0 International License, which permits use, sharing, adaptation, distribution and reproduction in any medium or format, as long as you give appropriate credit to the original author(s) and the source, provide a link to the Creative Commons licence, and indicate if changes were made. The images or other third party material in this article are included in the article's Creative Commons licence, unless indicated otherwise in a credit line to the material. If material is not included in the article's Cre- 
ative Commons licence and your intended use is not permitted by statutory regulation or exceeds the permitted use, you will need to obtain permission directly from the copyright holder. To view a copy of this licence, visit http://creativecommons.org/licenses/by/4.0/.

\section{REFERENCES}

1. Du Plessis, A. and Boshoff, W.P., A review of X-ray computed tomography of concrete and asphalt construction materials, Constr. Build. Mat., 2019, vol. 199, pp. 637-651. https://doi.org/10.1016/j.conbuildmat.2018.12.049

2. Brierley, N., Nye, B., and McGuinness, J., Mapping the spatial performance variability of an X-ray computed tomography inspection, NDT \& E Int., 2019, vol. 107, article ID: 102127. https://doi.org/10.1016/j.ndteint.2019.102127

3. Zikmund, T., Šalplachta, J., Zatočilová, A., Břínek, A., Pantělejev, L., Štěpánek, R., Koutný, D., Paloušek, D., and Kaiser, J., Computed tomography based procedure for reproducible porosity measurement of additive manufactured samples, NDT \& E Int., 2019, vol. 103, pp. 111-118. https://doi.org/10.1016/j.ndteint.2019.02.008

4. Du, Z., Hu, Y., Ali Buttar, N., and Mahmood, A., X-ray computed tomography for quality inspection of agricultural products: A review, Food Sci. Nutr., 2019, vol. 7, no. 10, pp. 3146-3160. https://doi.org/10.1002/fsn3.1179

5. Villarraga-Gómez, H., Thousand, J.D., and Smith, S.T., Empirical approaches to uncertainty analysis of X-ray computed tomography measurements: a review with examples, Precis. Eng., 2020, vol. 64, pp. 249-268. https://doi.org/10.1016/j.precisioneng.2020.03.004

6. Lüthi, M., Bircher, B.A., Meli, F., Küng, A., and Thalmann, R., X-ray flat-panel detector geometry correction to improve dimensional computed tomography measurements, Meas. Sci. Technol., 2019, vol. 31, no. 3, article ID: 035002. https://doi.org/10.1088/1361-6501/ab52b1

7. Körner, L., Lawes, S., Bate, D., Newton, L., Senin, N., and Leach, R., Increasing throughput in X-ray computed tomography measurement of surface topography using sinogram interpolation, Meas. Sci. Technol., 2019, vol. 30 , no. 12, article ID: 125002 .

https://doi.org/10.1088/1361-6501/ab37e5

8. Busi, M., Mohan, K.A., Dooraghi, A.A., Champley, K.M., Martz, H.E., and Olsen, U.L., Method for systemindependent material characterization from spectral X-ray CT, NDT \& E Int., 2019, vol. 107, article ID: 102136. https://doi.org/10.1016/j.ndteint.2019.102136

9. Fuchs, T. and Hanke, R. Task-driven design of X-ray systems for industrial inspection, 2008 IEEE Nucl. Sci. Symp. Conf. Record, 2008, pp. 523-527.

https://doi.org/10.1109/NSSMIC.2008.4775230

10. Yong, Y., Fei, L., Zhang, T., Chun-guang, X., Ming-chuan, Z., and Jing-rui, R., Design of synchronous projection data acquisition system for cone beam CT based on manipulator, 2016 IEEE Far East NDT New Technol. Appl. Forum (FENDT), 2016, pp. 190-194. https://doi.org/10.1109/FENDT.2016.7992022

11. Xiao, K., Han, Y., Xi, X., Yan, B., Bu, H., and Li, L., A parameter division based method for the geometrical calibration of X-ray industrial cone-beam CT, IEEE Access, 2018, vol. 6, pp. 48970-48977. https://doi.org/10.1109/ACCESS.2018.2865124

12. Hsieh, J., Computed tomography: Principles, design, artifacts, and recent advances, Bellingham : SPIE Press, 2015, 3rd ed.

13. Liu, X. and Lee, H.K., A simulation study of the spent nuclear fuel cask condition evaluation using high energy X-ray computed tomography, NDT \& E Int., 2016, vol. 80, pp. 58-64. https://doi.org/10.1016/j.ndteint.2016.02.008

14. Askari, M., Taheri, A., and Movafeghi, A., Industrial gamma computed tomography using high aspect ratio scintillator detectors (A Geant4 simulation), Nucl. Instrum. Methods Phys. Res., Sect. A, 2019, vol. 923, pp. 109-117. https://doi.org/10.1016/j.nima.2019.01.039

15. Hiller, J. and Reindl, L.M., A computer simulation platform for the estimation of measurement uncertainties in dimensional X-ray computed tomography, Measurement, 2012, vol. 45, no. 8, pp. 2166-2182. https://doi.org/10.1016/j.measurement.2012.05.030

16. Yang, Y., Wu, Y.C., Li, L., Zhang, S.Y., Dong, K.G., Zhang, T.K., Yu, M.H., Zhang, X.H., Zhu, B., Tan, F., Yan, Y.H., Li, G., Fan, W., Lu, F., Zhao, Z.Q., Zhou, W.M., Cao, L.F., and Gu, Y.Q., Design and characterization of high energy micro CT with a laser-based X-ray source, Results Phys., 2019, vol. 14, article ID: 102382. https://doi.org/10.1016/j.rinp.2019.102382

17. Peterzol, A., Létang, J.M., and Babot, D., A beam stop based correction procedure for high spatial frequency scatter in industrial cone-beam X-ray CT, Nucl. Instrum. Methods Phys. Res., Sect. B, 2008, vol. 266, no. 18, pp. 4042-4054. https://doi.org/10.1016/j.nimb.2008.07.005

18. Pan, X., Sidky, E.Y., and Vannier, M., Why do commercial CT scanners still employ traditional, filtered backprojection for image reconstruction?, Inverse Probl., 2009, vol. 25, no. 12, article ID: 123009.

https://doi.org/10.1088/0266-5611/25/12/123009 
19. Shi, L., Liu, B., Yu, H., Wei, C., Wei, L., Zeng, L., and Wang, G., Review of CT image reconstruction open source toolkits, J. X-Ray Sci. Technol., 2020, №. Preprint, pp. 1-22. https://doi.org/10.3233/XST-200666

20. Kengyelics, S.M., Treadgold, L.A., and Davies, A.G., X-ray system simulation software tools for radiology and radiography education, Comput. Biol. Med., 2018, vol. 93, pp. 175-183. https://doi.org/10.1016/j.compbiomed.2017.12.005

21. Sarantites, D.G. and Sobotka, L.G., Computational templates for introductory nuclear science using Mathcad, Am. J. Phys., 2013, vol. 81, no. 1, pp. 44-49. https://doi.org/10.1119/1.4764079

22. Duchesne, M.J., Moore, F., Long, B.F., and Labrie, J., A rapid method for converting medical Computed Tomography scanner topogram attenuation scale to Hounsfield Unit scale and to obtain relative density values, Eng. Geol., 2009, vol. 103, no. 3-4, pp. 100-105. https://doi.org/10.1016/j.enggeo.2008.06.009

23. Sakata, D., Haga, A., Kida, S., Imae, T., Takenaka, S., and Nakagawa, K., Effective atomic number estimation using kV-MV dual-energy source in LINAC, Physica Medica, 2017, vol. 39, pp. 9-15. https://doi.org/10.1016/j.ejmp.2017.06.010

24. Osipov, S.P., Chakhlov, S.V., Kairalapov, D.U., and Sirot'yan, E.V., Numerical modeling of radiographic images as the basis for correctly designing digital radiography systems of large-sized objects, Russ. J. Nondestr. Test., 2019, vol. 55, no. 2, pp. 136-149. https://doi.org/10.1134/S1061830919020050

25. Osipov, S.P., Yadrenkin, I.G., Chakhlov, S.V., Osipov, O.S., and Usachev, E.Yu., Simulation modelling in digital radiography with allowance for spatial outlines of test objects, Russ. J. Nondestr. Test., 2020, vol. 56, no. 8, pp. 647-660. https://doi.org/10.1134/S1061830920080082

26. Udod, V.A., Osipov, S.P., and Wang, Y., The mathematical model of image, generated by scanning digital radiography system, IOP Conf. Ser.: Mater. Sci. Eng., IOP Publ., 2017, vol. 168, no. 1, article no. 012042. https://doi.org/10.1088/1757-899X/168/1/012042

27. Ramachandran, G.N. and Lakshminarayanan, A.V., Three-dimensional reconstruction from radiographs and electron micrographs: application of convolutions instead of Fourier transforms, Proc. Natl. Acad. Sci., 1971, vol. 68 , no. 9 , pp. $2236-2240$.

https://dx.doi.org/10.1073\%2Fpnas.68.9.2236

28. Shepp, L.A. and Logan, B.F., The Fourier reconstruction of a head section, IEEE Trans. Nucl. Sci., 1974, vol. 21 , no. 3 , pp. $21-43$.

https://doi.org/10.1109/TNS.1974.6499235

29. NIST standard reference database 8 (XGAM). https://doi.org/10.18434/T48G6X. Accessed 2008.

30. Buratti, A., Grozmani, N., Voigtmann, C., Sartori, L.V., and Schmitt, R.H., Determination of the optimal imaging parameters in industrial computed tomography for dimensional measurements on monomaterial workpieces, Meas. Sci. Technol., 2018, vol. 29, no. 11, article ID: 115009.

https://doi.org/10.1088/1361-6501/aae4d6

31. Osipov, S., Chakhlov, S., Shan, J., and Kairalapov, D., Analysis of the possibility of determining the internal structure of oil and gas pipes by CT method, Mater. Sci. Forum., 2019, vol. 970, pp. 187-201. https://doi.org/10.4028/www.scientific.net/MSF.970.187

32. Mei, K., Ehn, S., Oechsner, M., Kopp, F.K., Pfeiffer, D., Fingerle, A.A., Pfeiffer, F., Combs, S.E., Wilkens, J.J., Rummeny, E.J., and Noël, P.B., Dual-layer spectral computed tomography: measuring relative electron density, Eur. Radiol. Exp., 2018, vol. 2, no. 1, article ID: 20. https://doi.org/10.1186/s41747-018-0051-8

33. Groenewald, A., and Groenewald, W.A., A universal phantom suitable for quality assurance on X-ray imaging modalities, Acta Radiologica, 2019, vol. 60, no. 11, pp. 1523-1531. https://doi.org/10.1177\%2F0284185119831685

34. Emre, T., Löffler, M., and Ehrenfried, Z., Deep learning-based inaccuracy compensation in reconstruction of high resolution XCT data, Sci. Rep., 2020, vol. 10, article ID: 7682. https://doi.org/10.1038/s41598-020-64733-7

35. Paziresh, M., Kingston, A.M., Latham, S.J., Fullagar, W.K., and Myers, G.M., Tomography of atomic number and density of materials using dual-energy imaging and the Alvarez and Macovski attenuation model, J. Appl. Phys., 2016, vol. 119, no. 21, pp. 214901-1-214901-11. https://doi.org/10.1063/1.4950807

36. Osipov, S., Chakhlov, S., Batranin, A., Osipov, O., and Kytmanov, J., Theoretical study of a simplified implementation model of a dual-energy technique for computed tomography, NDT \& E Int., 2018, vol. 98, pp. 63-69. https://doi.org/10.1016/j.ndteint.2018.04.010

37. Rizescu, C., Beşliu, C., and Jipa, A., Determination of local density and effective atomic number by the dualenergy computerized tomography method with the 192Ir radioisotope, Nucl. Instrum. Methods Phys. Res., Sect. A, 2001, vol. 465, no. 2-3, pp. 584-599.

https://doi.org/10.1016/S0168-9002(01)00181-4 